\title{
Efeito do treinamento físico específico nas respostas cardiorrespiratórias e metabólicas em repouso e no exercício máximo em jogadores de futebol profissional
}

\author{
Paulo Roberto Santos Silva ${ }^{1}$, Angela Romano ${ }^{1}$, Paulo Yazbek Jr. ${ }^{2}$ e Linamara Rizzo Battistella ${ }^{3}$ \\ Divisão de Medicina de Reabilitação do Hospital das Clínicas da Faculdade de \\ Medicina da Universidade de São Paulo - Laboratório de Fisiologia do Exercício
}

\begin{abstract}
RESUMO
O objetivo deste estudo foi analisar as alterações provocadas pelo treinamento físico específico (TFE) nas respostas cardiorrespiratórias e metabólicas de 16 jogadores de futebol profissional, com média de idade de $24,2 \pm 3,6$ anos. Todos os atletas foram avaliados antes e depois de quinze semanas de um programa de TFE, durante período competitivo. Os futebolistas foram submetidos a teste máximo em esteira rolante, utilizando-se o protocolo de Ellestad ${ }^{20}$. A resposta de frequiência cardíaca (FC) foi registrada por meio de um eletrocardiógrafo de 3 derivações simultâneas e a pressão arterial (PA), por meio de método auscultatório. A ventilação pulmonar $\left(\mathrm{V}_{\mathrm{E}}\right)$, o consumo de oxigênio $\left(\mathrm{VO}_{2}\right)$, a produção de dióxido de carbono $\left(\mathrm{VCO}_{2}\right)$ e a razão de troca respiratória (RER) foram calculados a partir de valores medidos por um sistema espirométrico computadorizado (Beckman) e a capacidade anaeróbia máxima, por meio da concentração sanguínea de ácido lático, utilizando-se analisador automático. O TFE não modificou significativamente a FC máxima (192 \pm 8 vs. $186 \pm 6$ bpm) e a PA sistólica máxi-
\end{abstract}

\footnotetext{
1. Fisiologista do exercício.

2. Cardiologista chefe do laboratório de fisiologia.

3. Fisiatra e Diretora da Divisão de Medicina de Reabilitação do HC-FMUSP.
}

Endereço para correspondência:

Autor

Paulo Roberto Santos Silva

Rua Frederico Bartholdi, 566 - Saúde

04193-000 - São Paulo, SP

Tel. (011) 6969-6481

Instituição

Divisão de Medicina de Reabilitação - HC-FMUSP

Laboratório de Fisiologia do Exercício

Rua Diderot, 43 - Vila Mariana

04116-030 - São Paulo, SP

Tel. (011) 549-0111/Fax (011) 549-0556 ma $(196 \pm 10$ vs. $198 \pm 8 \mathrm{mmHg})$. A resposta ventilatória máxima foi significativamente aumentada $(129 \pm 19$ vs. 140 $\left.\pm 16 \mathrm{~L} \cdot \mathrm{min}^{-1}[\mathrm{p}<0.05]\right)$, enquanto a capacidade aeróbia máxima não foi significativamente modificada $(50,0 \pm 6,0 \mathrm{vs}$. $53,0 \pm 5,0 \mathrm{ml} . \mathrm{kg} \cdot{ }^{-1} \mathrm{~min}^{-1}$ ) por esse treinamento. Ao contrário, a capacidade anaeróbia máxima aumentou significativamente $\left(8,3 \pm 0,2\right.$ vs. $\left.9,8 \pm 2,4 \mathrm{mmol} \cdot \mathrm{L}^{-1}[\mathrm{p}<0,05]\right)$. Concluiu-se: 1$)$ o TFE não modificou as respostas de FC e PA no repouso e no exercício máximo; 2) a maior $\mathrm{V}_{\mathrm{E}}$ no exercício máximo associada a elevada concentração sanguínea de ácido lático demonstraram que o TFE utilizado nesse estudo foi caracterizado por exercícios predominantemente intensos; e 3) o TFE não representou estímulo adequado para aumentar a capacidade aeróbia máxima dos futebolistas.

Palavras-chave: Treinamento físico. Capacidade aeróbia. Capacidade anaeróbia. Freqüência cardíaca. Pressão arterial. Jogador de futebol profissional. Medicina esportiva.

\section{ABSTRACT \\ Effect of specific exercise training in metabolic and car- diorespiratory responses at rest and maximum exercise in professional soccer players}

The purpose of this investigation was to study the effect of specific training on cardiorespiratory and metabolic responses in sixteen male professional soccer players, aged $24.2 \pm$ 3.6 years old. They were evaluated before and after fifteen weeks of specific training. All subjects were submitted to an exercise test on treadmill, using Ellestad's protocol. The heart rate was recorded by means of ECG, and the arterial blood pressure by auscultatory measurement. Oxygen uptake, carbon dioxide production, and pulmonary ventilation were measured by a Measurement Metabolic Cart (Beckman). Maximum anaerobic capacity was evaluated by blood lactate concentration, using an automated analyzer. Specific training did not modify the maximum heart rate (192 $\pm 8 v$ s. $186 \pm 6$ 
beats. $\left.\mathrm{min}^{-1}\right)$ and the maximum systolic blood pressure (196 \pm 10 vs. $198 \pm 8 \mathrm{mmHg}$ ). Maximum respiratory ventilation was significantly increased by specific training (129 \pm 19 vs. $140 \pm 16{\mathrm{~L} . \mathrm{min}^{-1}}^{-}$), whereas maximum aerobic capacity was not significantly increased $\left(50 \pm 6\right.$ vs. $53 \pm 5 \mathrm{ml} . \mathrm{kg}^{-1}$ $\mathrm{min}^{-1}$ of $\mathrm{O}_{2}$ ). On the other hand, maximum aerobic capacity improved significantly $\left(8.3 \pm 0.2 \mathrm{vs} .9 .8 \pm 2.4\right.$ mmol. $\left.\mathrm{L}^{-1}\right)$. Conclusion: 1) the specific training used in the present study did not modify heart rate and arterial blood pressure responses at rest and during maximum exercises; 2) improvement of maximum pulmonary ventilation and higher lactic acid concentrations during maximum exercise showed that the exercise training used in this study was predominantly characterized by intense exercise, and 3) specific training was not an adequate stimulus to improve maximum aerobic capacity of the soccer players.

Key words: Exercise training. Aerobic capacity. Anaerobic capacity. Heart rate. Blood pressure. Professional soccer player. Sports medicine.

\section{INTRODUÇÃO}

A ciência nas últimas décadas tem tido importante participação no futebol. Conseqüentemente, o nível do jogo e dos jogadores em muitos pormenores tem melhorado. Como em todos os esportes coletivos, o futebol é um jogo de campo complexo, com vários aspectos interativos, em que a eficiência dos jogadores durante as partidas depende principalmente da associação de fatores táticos, técnicos e físicos. Portanto, é importante conhecer e identificar características de variáveis funcionais que interferem no rendimento do futebolista, como também de indicadores que possam ser utilizados como referência para o treinamento desses atletas.

O futebol é um jogo de demanda energética multifatorial e sua variação é muito grande durante as partidas ${ }^{1,2}$. É uma modalidade esportiva extremamente popular e muito difundida em vários países. Entretanto, pouco se conhece a respeito das características fisiológicas de jogadores profissionais em nosso país. Além disso, as informações disponíveis são de jogadores amadores e profissionais de outros países ${ }^{3-}$ ${ }^{9}$, o que torna o jogador profissional brasileiro ainda menos conhecido $^{1,5,10-12}$.

Alguns pesquisadores têm concentrado seus estudos nas respostas fisiológicas agudas de freqüência cardíaca (FC), concentração sanguínea de ácido lático e capacidade aeróbia máxima ( $\mathrm{VO}_{2}$ máx.), sendo esta última considerada uma das mais importantes características para o sucesso nesse esporte. Por outro lado, é reconhecido que a importância de cada um desses parâmetros fisiológicos parece depender, em grande parte, da posição e das funções táticas durante o transcorrer do jogo, da motivação, nível de qualidade técnica, carga genética e do grau de aptidão física do jogador ${ }^{13-19}$.

\section{2}

Estudos longitudinais que demonstraram a evolução comparativa de respostas fisiológicas do treinamento físico específico (TFE), em meio à competição, em futebolistas profissionais são escassos ${ }^{1,2}$.

Este estudo teve por objetivo analisar o comportamento cardiorrespiratório e metabólico, em repouso e no exercício máximo, de um grupo de jogadores de futebol profissional, pertencentes à primeira divisão do Estado de São Paulo, antes do início do campeonato (pré-temporada) e após quinze semanas de um programa de TFE e competição.

\section{MATERIAL E MÉTODOS}

Dezesseis jogadores de futebol profissional do sexo masculino, com média de idade de $24,2 \pm 3,6$ anos, peso de 75,0 $\pm 8,0 \mathrm{~kg}$ e estatura de $179 \pm 5 \mathrm{~cm}$, pertencentes à primeira divisão do Estado de São Paulo, foram avaliados em período de pré-temporada e quinze semanas após o início do campeonato paulista, isto é, no período intermediário da competição (tabela 1).

\begin{tabular}{|c|c|c|c|}
\hline \multicolumn{4}{|c|}{$\begin{array}{c}\text { TABELA } 1 \\
\text { Características físicas dos } 16 \text { jogadores de futebol profissional } \\
\text { avaliados antes e após treinamento físico específico (TFE) }\end{array}$} \\
\hline Fase & $\begin{array}{l}\text { Idade } \\
\text { (anos) }\end{array}$ & $\begin{array}{c}\text { Peso } \\
\text { (kg) }\end{array}$ & $\begin{array}{l}\text { Estatura } \\
\text { (cm) }\end{array}$ \\
\hline Pré-TFE & $24,2 \pm 3,6$ & $75,0 \pm 8,0$ & $179 \pm 5$ \\
\hline Pós-TFE & $24,6 \pm 3,6$ & $74,0 \pm 7,0$ & $179 \pm 5$ \\
\hline
\end{tabular}

Resultados representam a média e o desvio-padrão.

Previamente à avaliação em esforço, cada jogador foi submetido a eletrocardiograma em repouso, com 12 derivações, registradas em eletrocardiógrafo Hewlett-Packard (modelo 7826-B), para diagnosticar possíveis alterações eletrocardiográficas. Além dessa medida, a pressão arterial foi avaliada por meio de método indireto auscultatório, utilizando-se esfigmomanômetro aneróide.

Antecedendo ao período de TFE, os atletas foram submetidos a um teste máximo, em esteira rolante (Quinton modelo 18-54), utilizando-se o protocolo de Ellestad $^{20}$.

A frequiência cardíaca (FC) foi monitorada continuamente em repouso, durante o teste de esforço e na fase de recuperação, por meio de um eletrocardiógrafo (Dixtal modelo DX2500) com 3 derivações simultâneas nas posições $\left(\mathrm{MC}_{5}\right.$, $\mathrm{AV}_{\mathrm{F}}$ e $\mathrm{V}_{2}$ ) e registrada a cada minuto. As pressões arteriais sistólica (PAS) e diastólica (PAD) em repouso e a PAS no final de cada estágio do teste de esforço foram medidas por meio de um esfigmomanômetro aneróide.

A ventilação pulmonar $\left(\mathrm{V}_{\mathrm{E}}\right)$, o consumo de oxigênio $\left(\mathrm{VO}_{2}\right)$, a produção de dióxido de carbono $\left(\mathrm{VCO}_{2}\right)$ e a razão de troca respiratória $\left(\mathrm{RER}\left[\mathrm{VCO}_{2} / \mathrm{VO}_{2}\right]\right.$ ) foram calculados a partir de valores medidos por meio de um analisador de gases compu- 
tadorizado (Beckman modelo MMC), que possui um sensor polarográfico (OM-11) e um sistema infravermelho (LB-2) para avaliação das frações expiradas de oxigênio $\left(\mathrm{FEO}_{2}\right)$ e de dióxido de carbono $\left(\mathrm{FECO}_{2}\right)$, respectivamente ${ }^{21,22}$.

A capacidade anaeróbia lática máxima foi determinada pela concentração sanguínea de ácido lático, por meio de amostras de $60 \mu \mathrm{l}$ de sangue arterializado, colhidas da polpa do dedo médio, seguido de análise em sistema automático (YSI L-Lactate modelo $23 \mathrm{~L}$ ). As determinações de ácido lático sanguíneo foram realizadas em repouso e no segundo minuto de recuperação.

O TFE realizado nesse período foi subdividido em duas fases. A primeira durou quatro semanas e denominou-se de pré-temporada. Nas duas primeiras semanas, o treinamento físico diário foi realizado em três períodos, com $60 \%$ dos exercícios sendo utilizados para a melhoria da capacidade cardiorrespiratória (base aeróbia) e os restantes $40 \%$ foram destinados para a melhoria da flexibilidade, resistência muscular localizada e treinamento técnico com bola, objetivando o aperfeiçoamento de passes, chutes, cabeceios, cruzamentos, dribles e outros. Nas duas últimas semanas, $70 \%$ do volume de treinamento foram direcionados para a intensidade, com o desenvolvimento da velocidade e resistência de velocidade, entremeados com treinamentos técnicos e táticos.

$\mathrm{Na}$ fase final da pré-temporada, diminuiu-se significativamente o estímulo aeróbio, com apenas um volume de $30 \%$ dos exercícios realizados para a melhoria dessa condição metabólica.

No período denominado competitivo, com dois jogos realizados por semana, os jogadores treinaram (físico, técnico e tático) em média 20 horas por semana, com ênfase dada aos exercícios de alta intensidade com e sem bola. A condição aeróbia recebeu pouca atenção nessa fase.

Os dados foram analisados por meio do teste $t$ de Student, fixando-se o nível de significância de $\mathrm{p}<0,05$, para dados $\operatorname{pareados}^{23}$.

\section{RESULTADOS}

Respostas cardiovasculares: a FC e as PAS e PAD em repouso não foram modificadas significativamente pelo TFE (tabela 2). Resultados semelhantes foram observados no exercício máximo, ou seja, o TFE não modificou a PAS na intensidade máxima de exercício (tabela 2).

Respostas respiratórias e metabólicas no exercício máximo: a $V_{E}$, na intensidade máxima de exercício foi significativamente aumentada após o TFE. Isso representou um aumento de $11 \mathrm{~L} \cdot \mathrm{min}^{-1}$ ou um ganho de $9 \%$, em conseqüência do TFE utilizado (tabela 3).

Resultados semelhantes foram verificados em relação à concentração sanguínea máxima de ácido lático que, após o TFE, apresentou aumento de $18 \%$ (tabela 3). Ao contrário, o
TABELA 2

Resultados de freqüência cardíaca (FC) e pressão arterial (PA) sistólica (PAS) e diastólica (PAD) em repouso e no exercício físico máximo antes e após treinamento físico específico (TFE)

\begin{tabular}{|c|c|c|c|c|c|}
\hline \multirow[b]{2}{*}{ Fase } & \multicolumn{3}{|c|}{ Repouso } & \multicolumn{2}{|c|}{ Exercício máximo } \\
\hline & $\begin{array}{c}\text { FC } \\
\text { (bpm) }\end{array}$ & $\begin{array}{c}\text { PAS } \\
(\mathrm{mmHg})\end{array}$ & $\begin{array}{c}\text { PAD } \\
(\mathrm{mmHg})\end{array}$ & $\begin{array}{c}\text { FC } \\
\text { (bpm) }\end{array}$ & $\begin{array}{c}\text { PAS } \\
(\mathrm{mmHg})\end{array}$ \\
\hline Pré-TFE & $66 \pm 8$ & $119 \pm 12$ & $78 \pm 6$ & $192 \pm 8$ & $196 \pm 10$ \\
\hline Pós-TFE & $63 \pm 11$ & $118 \pm 13$ & $76 \pm 11$ & $186 \pm 6$ & $198 \pm 8$ \\
\hline
\end{tabular}

Resultados representam a média e o desvio-padrão.

TABELA 3

Resultados de ventilação pulmonar $\left(V_{E}\right)$, consumo máximo de oxigênio ( $\mathrm{VO}_{2}$ máx) e ácido lático sanguíneo (AL) no exercício máximo antes e após treinamento físico específico (TFE)

\begin{tabular}{|c|c|c|c|}
\hline Fase & $\begin{array}{l}\text { VE }_{\text {BTPS }} \\
\left(\text { L.min }^{-1}\right)\end{array}$ & $\begin{array}{l}\text { VO } 2 \text { máx STPD } \\
\left(\mathrm{ml} . \mathrm{kg}^{-1} \cdot \mathrm{min}^{-1}\right)\end{array}$ & $\begin{array}{c}\text { AL } \\
\left(\mathrm{mmol} . \mathrm{L}^{-1}\right)\end{array}$ \\
\hline Pré-TFE & $129 \pm 19$ & $50,0 \pm 6,0$ & $8,3 \pm 0,2$ \\
\hline Pós-TFE & $140 \pm 16 *$ & $53,0 \pm 5,0$ & $9,8 \pm 2,4^{*}$ \\
\hline
\end{tabular}

Resultados representam a média e o desvio-padrão.

$*$ = Diferença significativa $p<0,05$ entre as fases pré e pós-TFE.

$\mathrm{VO}_{2}$ máx foi modificado em apenas $6 \%$ após o TFE (tabela $3)$.

\section{COMENTÁRIOS E DISCUSSÃO}

O principal objetivo deste estudo foi verificar as alterações cardiorrespiratórias e metabólicas provocadas pelo TFE, comumente realizado por jogadores de futebol em período competitivo.

Apesar de o treinamento físico provocar bradicardia de repouso, tanto no homem ${ }^{24-26}$ como no animal ${ }^{27-31}$, na presente investigação esse fenômeno não foi verificado. Essa distinção evidencia que o TFE realizado pelos futebolistas não foi direcionado para exercícios de intensidade predominantemente moderada e de longa duração, pois é a partir do exercício realizado com essas características que se verifica diminuição da FC de repouso. A resposta cronotrópica máxima de exercício também não foi modificada significativamente pelo TFE.

O efeito do treinamento físico na PA de repouso é contraditório ${ }^{32}$. Embora alguns autores sugiram que o treinamento físico diminui a PA de repouso, estes resultados não foram verificados no presente estudo.

A resposta ventilatória máxima verificada após o TFE foi significativamente aumentada. Resultados semelhantes foram encontrados por Raven et al. ${ }^{5}$, em jogadores de futebol profissional. Esse aumento na $V_{E}$ pode ter sido determinado pela elevada intensidade do TFE utilizado. Tem sido demons- 
trado que o treinamento físico intenso aumenta a capacidade ventilatória de exercício, como um mecanismo de compensação da acidose metabólica ${ }^{33-36}$.

A concentração sanguínea de ácido lático é, freqüentemente, utilizada como um indicador da produção de energia anaeróbia lática em futebolistas. A sua detecção tem sido realizada como rotina em laboratórios de fisiologia ou, mais especificamente, entre os períodos intermediário e final das partidas, o que demonstra de maneira mais real a participação desse metabólito como produtor de energia durante o transcorrer de um jogo de futebol ${ }^{1,2}$.

Gerisch et ll $^{37}$, medindo ácido lático em futebolistas amadores ao final das duas etapas do jogo, verificaram no transcorrer de quatro jogos competitivos valores de 5,58 e 4,68 mmol.L $\mathrm{L}^{-1}$, taxas consideradas baixas, quando comparadas a atletas que solicitam eficientemente essa via metabólica. Entretanto, esses resultados devem ser analisados com cuidado, pois os jogadores eram amadores e jovens. Resultados semelhantes foram encontrados por Rhode \& Espersen ${ }^{38} \mathrm{e}$ Smith et al. $^{39}$, em jogadores dinamarqueses e colegiais ingleses.

Ekblom $^{1}$, num estudo de grande profundidade, examinou várias divisões de categoria em jogadores de futebol da liga sueca adulta e constatou que a maior capacidade anaeróbia máxima foi verificada nas divisões mais altas. Essa constatação feita por Ekblom ${ }^{1}$ veio confirmar os resultados de Nowacki et al. ${ }^{8}$, que, testando os jogadores da seleção alemã de futebol, que disputou a Copa do Mundo em 1982, verificaram valor médio de ácido lático de $12,3 \mathrm{mmol}^{-1} \mathrm{~L}^{-1}$, sendo que um futebolista atingiu 16,2 mmol.L ${ }^{-1}$.

Em nosso estudo, a maior concentração sanguínea de ácido lático (18\%), no exercício máximo, demonstrou que o TFE representou um estímulo eficiente para o aumento da capacidade anaeróbia dos jogadores. No entanto, há de se ressaltar uma possível predisposição dos futebolistas em assimilar esse tipo de treinamento, conforme demonstraram Jacobs et al. ${ }^{40}$ em estudo sobre a distribuição de fibras musculares esqueléticas em futebolistas. Os jogadores de futebol profissional apresentaram, proporcionalmente, maior número de fibras de contração rápida do tipo II (anaeróbias) em relação às fibras de contração lenta do tipo I (aeróbias), o que representa uma importante característica fisiológica para a assimilação de um treinamento físico anaeróbio. Entretanto, mais recentemente, Kuzon et al. ${ }^{41}$, estudando futebolistas de alto nível por meio de biópsia músculo-esquelética, verificaram hipertrofia das fibras musculares dos tipos I e II, indicando simultaneamente a capacidade de adaptação em ambas as fibras, durante os treinamentos aeróbio e anaeróbio, respectivamente.

Todavia, Parente et al. ${ }^{42}$, estudando trinta jogadores de futebol, também por biópsia muscular, verificaram que a posição adotada em jogo foi um fator preponderante para a maior utilização de um determinado tipo de fibra muscular. No estudo deles, as fibras vermelhas de contração lenta (aeróbias) do tipo I foram encontradas em maior proporção nos jogadores de meio-campo (67\%); ao contrário, as fibras IIa de contração intermediária (aeróbia/anaeróbia) e as fibras brancas IIb (anaeróbias) de contração rápida foram nesses jogadores de somente 16 e $17 \%$, respectivamente. Os zagueiros e atacantes demonstraram maior equilíbrio das fibras do tipo I (aeróbias) e IIb (anaeróbias), com percentagens de 44 e $49 \%$ e 38 e 40\%, respectivamente. Enquanto isso, as fibras IIa (intermediárias) foram somente de 7 e $22 \%$ em jogadores dessas posições. Miller ${ }^{43}$ mostra algumas das características morfológicas, fisiológicas e bioquímicas desses tipos de fibras no músculo esquelético (ver quadro 1).

QUADRO 1

Características morfológicas, fisiológicas e bioquímicas das fibras no músculo esquelético (Miller, 1992)

Característica

1) Tamanho do neurônio motor

2) Velocidade de condução do neurônio motor

3) Tempo de contração

4) Produção de força

5) Elasticidade

6) Densidade mitocondrial

7) Concentração de mioglobina

8) Resistência a fadiga

9) Atividade de enzimas anaeróbias

10) Atividade de enzimas aeróbias

11) Sistema energético predominante

12) Estoques de fosfocreatina

13) Estoques de glicogênio

14) Atividade da miosina ATPase

Fibra tipo I
(vermelha) lenta
(aeróbia)

Pequeno
Lenta
Lento
Baixa
Baixa
Alta
Alta
Alta
Baixa
Alta
Aeróbio
Baixo
Baixo
Baixa

Fibra lla
intermediária
(aeróbia/anaeróbia)

Grande
Rápida
Rápido
Alta
Alta
Alta
Média
Baixa
Alta
Alta
Aeróbio/anaeróbio
Alto
Alto
Alta

\section{Fibra Illb (branca) rápida (anaeróbia)}

Grande

Rápida

Rápido

Alta

Alta

Baixa

Baixa

Baixa

Alta

Baixa

Anaeróbio

Alto

Alto

Alta 
Contudo, ainda não está claro se essas diferenças são motivadas por estímulos que podem estar relacionados a fatores como: comportamento do jogador durante a partida, característica do jogo, motivação, morfologia muscular e estratégia tática.

Entretanto, a produção de energia anaeróbia lática é considerada um fator importante, pois o jogador com elevada capacidade anaeróbia estará melhor preparado para realizar exercício de alta intensidade, durante períodos da partida ${ }^{37}$.

Por outro lado, o consumo máximo de oxigênio ( $\mathrm{VO}_{2}$ máx.) não foi significativamente alterado pelo TFE. Esses resultados reforçam a interpretação anterior de que o TFE empregado no presente estudo representou, por si só, pouco estímulo para aumentar a capacidade oxidativa máxima. Nessa investigação, $\mathrm{o} \mathrm{VO}_{2}$ máx. aumentou apenas $6 \%$ (não significativo), após quinze semanas de TFE, o que contrasta com o aumento significativo de 15\% verificado por Fardy ${ }^{44}$ em jogadores amadores, após cinco semanas de treinamento.

Entretanto, resultados semelhantes aos nossos foram observados por $\mathrm{Bangsbo}^{2} \mathrm{em}$ jogadores de futebol dinamarqueses. Ele verificou um aumento de apenas $3 \%$ no $\mathrm{VO}_{2}$ máx. após cinco semanas de treinamento. Ao contrário, o tempo de tolerância a esforços de alta intensidade aumentou $10 \%$, demonstrando que nesse período o treinamento físico aplicado desenvolveu-se através de exercícios predominantemente intensos. Além disso, é importante ressaltar que os resultados de $\mathrm{VO}_{2}$ máx. obtidos em nosso laboratório são consistentemente inferiores aos de jogadores amadores e profissionais verificados por outros autores $1,2,8,9,18,45-49$.

A capacidade aeróbia máxima é considerada um fator importante para o sucesso de jogadores de futebol, pois alguns relatos $^{33,50}$ demonstraram que futebolistas de alto nível solicitam o metabolismo aeróbio em mais de $80 \%$ do máximo durante o jogo. Além disso, quando o jogo é realizado em condições normais de temperatura, há diminuição do peso corpóreo de aproximadamente $2 \mathrm{~kg}$, o que mais uma vez sugere elevada demanda de energia proveniente desse sistema metabólico ${ }^{2}$.

Níveis elevados de $\mathrm{VO}_{2}$ máx. exercem também papel secundário importante na recuperação mais rápida da energia proveniente do sistema alático (ATP-CP), responsável por considerável fornecimento de energia durante períodos de alta intensidade como também na remoção mais eficiente do ácido lático nos momentos de repouso ativo e/ou diminuição da intensidade do exercício durante o jogo $0^{51-54}$.

Ekblom $^{1}$, estudando esse parâmetro há mais de 20 anos, tem observado a sua crescente evolução em futebolistas. Entretanto, na literatura especializada não encontramos um padrão de referência absoluto para valores de $\mathrm{VO}_{2}$ máx. em futebolistas; ao contrário, verifica-se grande variação em sua detecção ${ }^{55,56}$. Além disso, tem sido discutido se essa variação está relacionada a aspectos como: nível de qualidade técnica, motivação, estratégia tática com funções e/ou posições ocupadas em campo pelos jogadores, efeito de treinamento, carga genética ou até mesmo todos juntos ${ }^{13-18,37,57}$.

Segundo alguns autores ${ }^{1,58,59}$, valores de $\mathrm{VO}_{2}$ máx. entre 65 e $67 \mathrm{ml} . \mathrm{kg} .{ }^{-1} \mathrm{~min}^{1}$ parecem ser ideais para o futebolista correr eficientemente durante os 90min. de jogo. Entretanto, Nowacki ${ }^{60}$ concorda que desenvolver a capacidade aeróbia máxima em jogadores de futebol é de grande importância, mas, em sua opinião, valores acima de $70 \mathrm{ml} . \mathrm{kg} \cdot{ }^{-1} \mathrm{~min}^{-1}$ ou em níveis extremos $\left(85 \mathrm{ml} . \mathrm{kg} .{ }^{-1} \mathrm{~min}^{1}\right)$ tornam-se perigosos, pois podem comprometer a velocidade e a técnica (especificidade), qualidades muito importantes para o futebolista. O grupo de Bunc ${ }^{12}$, baseado em seus estudos e comparações feitas com resultados verificados na literatura especializada, acredita que a potência aeróbia em futebolista profissional de sucesso internacional tem que ser superior a $62 \mathrm{ml} \cdot \mathrm{kg}^{-1} \cdot \mathrm{min}^{-1}$.

Há um consenso geral de que o futebol atual mudou suas características, quando comparado a décadas passadas. $\mathrm{Na}$ atualidade, verifica-se maior grau de intensidade e volume de esforço realizado pelos jogadores ao final das partidas, o que seguramente tem modificado o padrão de solicitação física sobre os atletas. Portanto, é plenamente justificável a preocupação com o desenvolvimento adequado de uma elevada capacidade aeróbia máxima em futebolistas.

Entretanto, no Brasil, os calendários apertados e os compromissos dos clubes, muitas vezes, por falta de tempo adequado, dificultam o trabalho do preparador físico na realização de preparação mais qualitativa. Portanto, é comum a verificação de índices baixos em determinadas qualidades físicas. É importante salientar que as prioridades no futebol em nosso país são dependentes dos resultados atingidos pelo clube no transcorrer da competição. Sendo assim, a preparação física, às vezes, é colocada em plano secundário em detrimento do desenvolvimento técnico e tático da equipe. Além do que, é necessária uma reflexão mais profunda sobre a função e/ou funções exercida pelo futebolista e os índices de aptidão atlética mais adequados para o melhor desempenho do atleta durante o jogo.

Os resultados de $\mathrm{VO}_{2}$ máx. verificados no presente estudo foram inferiores aos de jogadores juniores ${ }^{6,8,46,61}$ e profissionais ${ }^{1,2,5,8,10,18,56}$ de diversos países, o que nos faz pensar que no período o TFE não se direcionou para o aumento da eficiência do sistema transportador de oxigênio dos jogadores.

Contudo, esses resultados não devem ser extrapolados para outras situações ou equipes, pois algumas limitações metodológicas não puderam ser eliminadas do estudo. A falta de um grupo-controle e, portanto, de um planejamento experimental, o desconhecimento da intensidade dos exercícios físicos realizados nesse período, a característica dos jogos e dos jogadores são fatores que não permitem uma interpretação mais objetiva dos resultados. Entretanto, é importante enfatizar que os resultados observados devem servir de alerta e até mesmo de reflexão para os preparadores físicos sobre a importância de um planejamento adequado, procuran- 
do desenvolver harmonicamente as qualidades físicas necessárias aos futebolistas, nas suas diversas fases de treinamento e competições.

É importante ressaltar que a supercompensação é o objetivo em qualquer programa de treinamento físico, pois é através dessa resposta que se verifica o aumento da aptidão física. Entretanto, ela depende de como cada processo orgânico se relaciona com os outros e como essa inter-relação é afetada pelo treinamento. Deve ser lembrado que temos um limite biológico para a sua manifestação, pois sempre atingimos um ponto em que a intensidade não pode ser mais aumentada; e se ocorrer, o desequilíbrio homeostático é desenvolvido. As conseqüências geradas são drásticas e se manifestam de várias formas, entre elas: lesões musculares, alterações do humor, falta de apetite, alterações fisiológicas, etc. Apesar das limitações citadas anteriormente e desse estudo terse restringido a avaliações padronizadas no período pré-temporada e após quinze semanas de TFE e jogos competitivos, os resultados permitem sugerir que: 1) o programa de TFE e competições realizados nesse período não modificaram as respostas de FC e PA em repouso e no exercício máximo; 2) a maior ventilação pulmonar no exercício máximo e a elevada concentração sanguínea de ácido lático demonstraram que as atividades físicas desenvolvidas foram caracterizadas por exercícios de alta intensidade e 3) o TFE e os jogos não apresentaram um estímulo adequado para aumentar a capacidade aeróbia máxima dos jogadores.

\section{REFERÊNCIAS}

1. Ekblom B. Applied physiology of soccer. Sports Medicine 1986;3:5060

2. Bangsbo J. The physiology of soccer - with special reference to intense intermittent exercise. Acta Physiol Scand 1994;151 Suppl:619.

3. Caru B, Le Coultre L, Aghemo P, Pinera-Limas F. Maximal aerobic and anaerobic muscular power in football players. J Sports Med 1970;10: 100-3.

4. Bell E, Rhodes G. The morphological characteristics of the association football player. Br J Sports Med 1975;9:196-200.

5. Raven PB, Gettman LR, Pollock ML. A physiological evaluation of professional soccer players. Br J Sports Med 1976;10:209-16.

6. Berg KE, La Voie JC, Latin RW. Physiological training effects of playing youth soccer. Med Sci Sports Exerc 1985;17:656-60.

7. Ramadan J, Bird R. Physical characteristics of elite soccer players. J Sports Med 1987;27: 424-8.

8. Nowacki PE, Cai DY, Buhl C, Krummelbein U. Biological performance of German soccer players (professionals and juniors) tested by special ergometry and treadmill methods. In: Reilly T, Lees A, Davis K, Murphy WJ, editors. Science and Football. Proceedings of the First World Congress of Science and Football, Liverpool 13-17th, April. 1987:145-57.

9. Chin MK, So RCH, Yuan YWY, Li RCT, Wong ASK. Cardiorespiratory fitness and isokinetic muscle strength of elite Asian junior soccer players. J Sports Med Phys Fitness 1994;34:250-7.

10. Williams C, Reid RM. Observation on the aerobic power of University rugby players and professional soccer players. Br J Sports Med 1973; 7:390-1.
11. Thomas V, Reilly T. Fitness assessment of English league soccer players through the competitive season. Br J Sports Med 1979;13:103-9.

12. Bunc V, Heller J, Prochazka L. Physiological characteristics of elite Czechoslovakia footballers. In: Communication to the Second World Congress on Science and Football. Held in Eindhoven, The Netherlands, p. 149; 22-25, May 1991. J Sports Sci 1992;10:139-205.

13. Klissouras V. Hereditability of adaptive variation. J Appl Physiol 1971; $31: 338-44$

14. Klissouras V. Prediction of athletic performance: genetic considerations. Can J Appl Spt Sci 1976;1:195-200.

15. Rochcongar P, Dassonville J, Lessardy Y. Consommation maximale d'oxygéne, lactacidémie et football. Medicine du Sport 1981;55:5-8.

16. Bouchard C, Lortie G. Heredity and endurance performance. Sports Med 1984;1:38-64.

17. Bouchard C, Lesage R, Lortie G. Aerobic performance in brothers, dizygotic and monozygotic twins. Med Sci Sports Exerc 1986;18:639-46.

18. Apor P. Successful formulae for fitness training. In: Reilly T, Lees A, Davis K, Murphy WJ, editors. Science and football. London/New York: E. \& F.N. Spon, 1988:95-107.

19. Puga N, Ramos J, Agostinho J, Lomba I, Costa O. Physiological profile of a 1st division Portuguese professional football team. In: Reilly, et al, editors. Science and Football II. Spon London: E \& F.N., 1993:40-2.

20. Ellestad MH. Maximal treadmill stress testing for cardiovascular evaluation. Circulation 1969;39:517-22.

21. Wilmore JH, Davis JA, Norton AC. An automated system for assessing metabolic and respiratory function during exercise. J Appl Physiol 1976; 40:619-24.

22. Yazbek Jr P, Camargo Jr PA, Kedor HH, Saraiva JF, Serro-Azul LG. Aspectos propedêuticos no uso da ergoespirometria. Arq Bras Cardiol 1985;44:291-5.

23. Glantz SA. Primer of Biostatistics. 3a ed. New York, Mc Graw-Hill, 1992.

24. Raab W, Silva PP, Marchet H, Kimura E, Starcheska YK. Cardiac adrenergic preponderance due to lack of physical exercise and its pathogenic implications. Am J Cardiol 1960;5:300-20.

25. Lin Y, Horvath SM. Autonomic nervous control of cardiac frequency in the exercise-training rat. J Appl Physiol 1972;33:796-9.

26. Katona PG, Mc Lean M, Dighton DH, Guz A. Sympathetic and parasympathetic cardiac control in athletes and nonathletes at rest. J Appl Physiol 1982;52:1652-7.

27. Tipton CM, Taylor B. Influence of atropine on the heart rates of rates of rats. Am J Physiol 1965;208:480-4.

28. Tipton CM. Training and bradycardia in rats. Am J Physiol 1965;209: 1089-94.

29. Tipton CM. Resting heart rate investigations with trained and nontrained hypophysectomized rats. J Appl Physiol 1969;26:585-8.

30. Sigvardsson K, Svanfeldt E, Kilbom A. Role of the adrenergic nervous system in development of training-induced bradycardia. Acta Physiol Scand 1977;101:481-8.

31. Gallo Jr L, Maciel BC, Marin-Neto JA, Martins LEB. Sympathetic changes in heart rate control during dynamic exercise induced by endurance training in man. J Med Biol Res 1989;22: 631-43.

32. Seals DR, Hagberg JM. The effect of exercise training on human hipertension: a review. Med Sci Sports Exerc 1984;16:207-15.

33. Astrand I, Astrand PO, Christensen EH, Hedman R. Intermittent muscular work. Acta Physiol Scand 1960;48:448-53.

34. Wasserman K, Whipp BJ, Koyal SN, Beaver WL. Anaerobic threshold and respiratory gas exchange during exercise. J Appl Physiol 1973;35: 236-43. 
35. Davis JA. Anaerobic threshold: review of the concept and directions for future research. Med Sci Sports Exerc 1985;17: 6-18.

36. Davis JA, Vodak P, Wilmore JH, Vodak J, Kurtz P. Anaerobic threshold alteration caused by endurance training in middle-aged men. J Appl Physiol 1976;46:1039-46.

37. Gerisch G, Rutemoller E, Weber K. Sportsmedical measurements of performance in soccer. In: Reilly T, Lees A, Davis K, Murphy WJ, editors. Science and football. Proceedings of the First World Congress of Science and Football, Liverpool 13-17th, April 1987:60-7.

38. Rhode HC, Espersen T. Work intensity during soccer training and matchplay. In: Reilly T, Leis A, Davis K, Murphy WJ, editors. Science and football. London: E. \& F.N. Spon 1988:68-75.

39. Smith M, Clarke G, Hale T, Mc Morris T. Blood lactate levels in college soccer players during match-play. In: Reilly T, Clarys J, Stibbe A, editors. Science and football II. London: E. \& F.N. Spon 1993:129-34.

40. Jacobs I, Westlin N, Karlsson J, Rasmusson M, Houghton B. Muscle glycogen and diet in elite soccer players. Eur J Appl Physiol 1982;48: 297-302.

41. Kuzon Jr WM, Rosenblatt JD, Huebel SC, Leatt P, Plyley MJ, McKee $\mathrm{NH}$, et al. Skeletal muscle fiber type, fibre size, and capillary supply in elite soccer players. Int J Sports Med 1990;11:99-102.

42. Parente C, Montagnani S, De Nicola A, Tajana GF. Anthropometric and morphological characteristics of soccer players according to positional role. In: Communications to the Second World Congress on Science and Football. Held in Eindhoven, The Netherlands, 22-25 May 1991. J Sports Science 1992;10:139-205.

43. Miller WC. The biochemistry of exercise and metabolic adaptations. Ed. Brown \& Benchmark, 1992:p.15.

44. Fardy PS. Effects of soccer training and detraining upon selected cardiac and metabolic measures. Res Q Exerc Sport 1969;40:503-8.

45. Rhodes EC, Mosher RE, Mc Kenzie DC, Franks IM, Potts JE, Wenger HA. Physiological profiles of the Canadian olympic soccer team. Can J Appl Sports Sci 1986;11:31-6.

46. Chin MK, Lo YS, Li CT, So CH. Physiological profiles of Hong-Kong elite soccer players. Br J Sports Med 1992;26:262-6.

47. Verma SK, Mohindroo SR, Kansal DK. The maximal anaerobic power of different categories of players. J Sports Med 1979;19:55-61.
48. Whiters RT, Roberts RGD, Davies GJ. The maximum aerobic power, anaerobic power and body composition of South Australian male representatives in athletics, basketball, field hockey and soccer. J Sports Med Phys Fitness 1977;17:391-400.

49. Leatt P, Shephard RJ, Plyley MJ. Specific muscular development in under-18 soccer players. J Sports Sci 1987;165-75.

50. Saltin B, Hermansen L. Esophageal, rectal and muscle temperature during exercise. J Appl Physiol 1966;21:1757-62.

51. Donovan CM, Brooks GA. Endurance training affects lactate clearance, not lactate production. Am J Physiol 1983;244:E83-E92.

52. Donovan CM, Pagliassotti MJ. Endurance training enhances lactate clearance during hyperlactatemia. Am J Physiol 1989;257:E782-E89.

53. Donovan CM, Pagliassotti MJ. Enhance efficiency of lactate removal after endurance training. J Appl Physiol 1990;68:1053-8.

54. Mac Era HSH, Dennis SC, Bosch AN, Noakes TD. Effects of training in lactate production and removal during progressive exercise in humans. J Appl Physiol 1992;72:1649-56.

55. Losada A. Clinical and instrumental profile of the cardiocirculatory system of a football player. In: Vecchiet L, editor. First Congress on Sports Medicine applied to Football. Proceedings I e II Roma. 1980:103-12.

56. Eclache JP, Viret R, Quard P, Ferret JM. Analyse de quelques donnés biometriques et energetiques de footballeurs professionnels Lyonnais. Cinesiologie 1981;20:64-99.

57. Van Gool D (1987). De fysieke belasting tijdens ean voetbalvedsfrifd: Studie van afgelegde afstand, hartfrequintie, energieverbruck en lactaatbapalingen. Thesis, University of Leuven, Leuven, Netherland.

58. Rost R, Hollmann W. Athlete's heart, a review of its historical assessments and new aspects. Int J Sports Med 1983;147-65.

59. Vanfraechem JHP, Thomas M. Maximal aerobic power and ventilatory threshold of a top level soccer team. Communication to the Second World Congress on Science and Football. J Sports Science 1992;10:139-205.

60. Nowacki PE. Die spiroergometrie in neun untersuchuengssystem fur den spitzensport. Leistungssport 1971;2:37-51.

61. Jones A, Helms P. Cardiorespiratory fitness in young British soccer players. J Sports Sci 1992;10:156-60. 